\title{
COMPARATIVE STUDY BETWEEN EPITHELIUM ON AND EPITHELIUM OFF COLLAGEN CROSS LINKING WITH RIBOFLAVIN AND UVR IN TREATMENT OF EARLY KERATOCONUS
}

\author{
Lotfy, M. ${ }^{(*)}$, Abd El-Badie, M. \& Youssef, H. \\ Ophthalmology dept., Faculty of Medicine, AL-Azhar Univ., Assuit, Egypt \\ *E-mail: mostafalotfi1987@yahoo.com
}

\begin{abstract}
Objective: To compare the safety and efficacy epithelium on versus epithelium off collagen cross linking with riboflavin and UVR in treatment of early keratoconus. Patients and Methods: The present study was a prospective, randomized study. 40 eyes from 25 patients with a mean age of 24 years (range: 15-35 years) were included. There were 15 males and 10 females, and all of them underwent corneal CXL using riboflavin and ultraviolet-A light at the Tipa Eye Hospital, Assuit. Results: Among the included samples, 20 eyes underwent epithelium-on corneal collagen CXL, and 20 eyes underwent epithelium-off corneal CXL. The incidence of postoperative pain was lowered by about $20 \%$ in the trans-epithelial group as compared with the epithelial-off group in the first postoperative week. In addition, the incidence of postoperative corneal haze was lowered by about $50 \%$ in the transepithelial group as compared with the epithelial-off group in the first postoperative week. It was also noticed that the CXL was formed in the upper-third of the corneal stroma, 20-30 $\mu \mathrm{m}$ beneath bowman membrane in the transepithelial CXL technique, while in the classic debridement technique; it was formed much deeper in the stoma. In group I, after 3 and 6 months, there was a non-statistically significant corneal thinning. In group II, none statistically significant corneal thinning was detected after 3 months, but after 6 months there was some regression in the form of corneal thinning by nearly 5-10 $\mu \mathrm{m}$ in $20 \%$ of cases. Conclusion: Both epithelium-off CXL and the epitheliumon CXL were effective in stabilizing the progression of keratoconus at least for 6 months; however, the epithelium-off CXL appeared to be much more effective in improving the refractive best-corrected visual acuity and topographic outcomes in keratoconic eyes compared with the epithelium-on CXL.
\end{abstract}

Keywords: Epithelium on CXL, Epithelium off CXL, Transepithelial CXL, Keratoconus, corneal cross linking, Riboflavin

\section{Introduction}

Cross-linking (CXL) of human collagen is a physiological process. Corneal CXL with ultraviolet-A (UVA) light is a new recent technique for enhancing the mechanical and chemical stability of the corneal tissue. The primary objective of this therapy is to produce extra chemical bonds within the corneal stroma through extremely localized photo polymerization while minimizing exposure to the surrounding eye structure [1]. The mechanism relies mainly on the action of free radicals and ends by increasing the covalent bonding between collagen fibrils. These results in 
several biomechanical, biochemical, and cellular changes in the corneal stroma, such as increased corneal stromal stiffness, increase in the collagen fibril diameter, more resistance to enzymatic digestion, and more resistance to hydrothermal shrinkage denoting greater stability $[2,3]$. There are different techniques used for corneal collagen CXL such as the following: Epithelium-off (Dresden protocol); This standard CXL

\section{Patients and Methods}

This study was a single-center, prospective, interventional study. It was conducted between December 2014 and May 2015, and included 40 eyes of 25 patients with a mean age 24 years (range: 15-33 years). There were fifteen males and ten females who underwent collagen CXL using riboflavin and UVA light at the Tipa Eye Hospital, Assuit. Written informed consent was obtained from all participants or from a legally responsible person after approval from the Institutional Ethics Committee. Keratoconus patients with history of progression over the previous 12 months (based on clinical and topographic history given by the referring doctor)

\subsection{Preoperative evaluation of patients}

A complete ocular examination was performed including visual acuity (VA) measurement using the 'Snellen chart', uncorrected (UCVA) and best spectacle corrected visual acuity (BSCVA) evaluation by the Decimal Visual Acuity Scale, anterior segment slit-lamp examination and ocular tension measurement. Both manifest and cycloplegic refraction were also assessed with cycloplegic refraction assessment $1 \mathrm{~h}$ after administration of $1 \%$ cyclopentolate eye drops. In addition, indirect ophthal- technique, also called the Dresden protocol, requires the removal of $9 \mathrm{~mm}$ from the center of the corneal epithelial layer (epioff), followed by $30 \mathrm{~min}$ of riboflavin administration, and subsequent application of UVA light for $30 \mathrm{~min}$. Transepithelial crosslinking; in the transepithelial epitheliumon (epi-on) CXL technique, the corneal epithelium layer is left intact [4].

were enrolled into this study. Patients who aged between 14 and 35 years and were able to complete all study visits were eligible for study enrolment. In addition, patients with Maximum K-reading less than $54 \mathrm{D}$ with Pentacam (OCULUS Optikgeräte $\mathrm{GmbH}$, Wetzlar, Germany) and Minimum Pentacam pachymetry greater than $400 \mu \mathrm{m}$, were also included. Patients with corneal thickness less than $400 \mu \mathrm{m}$, previous corneal surgery, significant central corneal opacity or scarring, other corneal disease, Systemic collagen vascular disease were excluded from the current study. Also, Women who are pregnant or nursing at the time of the initial treatment were not enrolled. moscopy to assess peripheral and the central part of the retina and Pentacam Pachymetry to assess the thickness of the central and peripheral cornea were carried out. Pentacam keratometry and corneal topography was performed using printouts of four refractive maps - the sagittal curvature map, corneal thickness map, and anterior and posterior elevation maps, fig. (1). Finally, anterior segment optical coherence tomography was performed to evaluate penetration of riboflavin into the stroma.

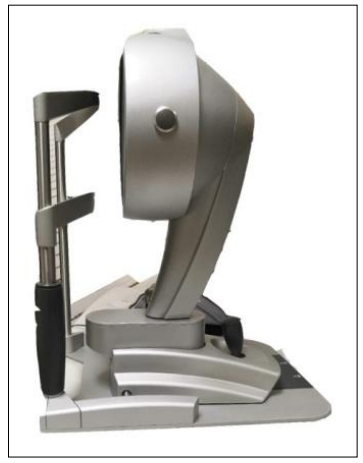




\subsection{Operative procedure}

\subsubsection{Group}

Group I included 20 eyes of 13 patients. The preoperative $\mathrm{K}$ max ranged between 47.2 and $52.6 \mathrm{D}$, and astigmatism ranged between -1.2 and $-8.0 \mathrm{D}$. All eyes underwent photo-oxidative CXL using riboflavin and UVA light after epithelial debridement of the central 8-9 $\mathrm{mm}$ of the cornea. All procedures were performed under topical anesthesia, administered twice for $2 \mathrm{~min}$ before the procedure, fig. (2-a, $\mathrm{b}, \mathrm{c})$. The epithelium was removed from the central 8-9 $\mathrm{mm}$ with a blunt metal spatula, fig. (2-a). De-epithelialization was followed by administration of riboflavin $(0.1 \%$ solu- tion $10 \mathrm{mg}$ riboflavin-5-phosphate in $10 \mathrm{ml}$ dextran-T- $50020 \%$ solution) every $3 \mathrm{~min}$ for 20-30 min until the stroma was completely penetrated and the aqueous was stained yellow, fig. (2-c). UVA irradiation was carried out using an optical system (UVX) illumination system version 1000; IROCAG, Zürich, Switzerland) with a light source composed of a range of UV di odes $(365 \mathrm{~nm})$ in combination with a pot potentiometer for voltage regulation. At the end of the procedure, a bandage soft contact lens was kept in place until full corneal re-epithelialization occurred.
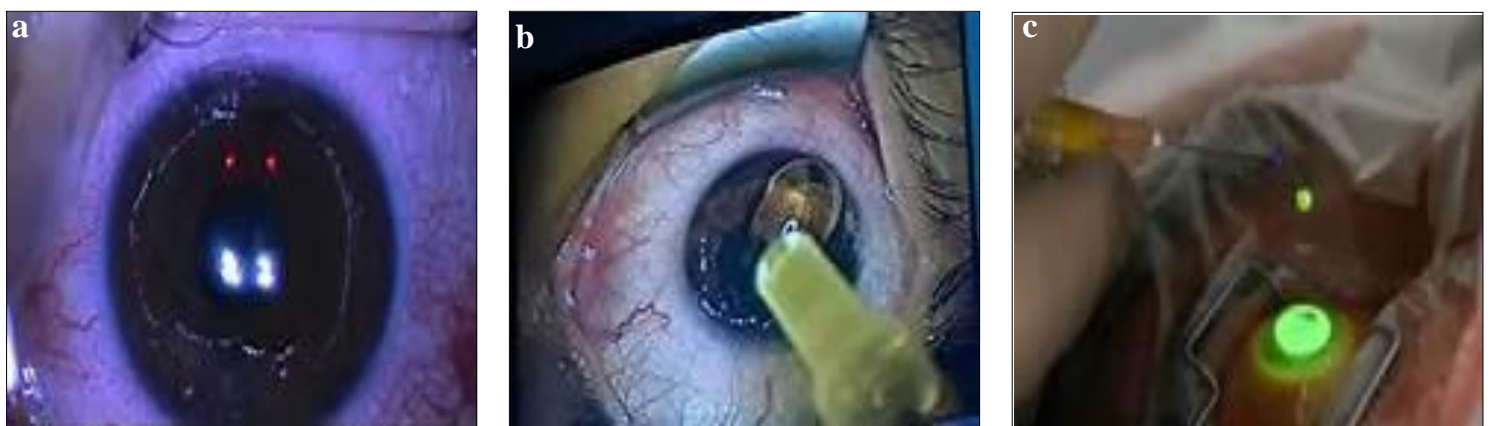

Figure (2) a. . removal of central $8 \mathrm{ml}$ corneal zone, $\underline{\mathbf{b}}$. application of riboflavin, $\underline{\mathbf{c}}$. application of riboflavin during use of UVA.

\subsubsection{Group II}

This group included 20 eyes of 12 patients. The preoperative $\mathrm{K}$ max ranged between 47.2 and $52 \mathrm{D}$ and astigmatism between -2.7 and $-8.3 \mathrm{D}$. Transepithelial CXL was performed on all 20 eyes where

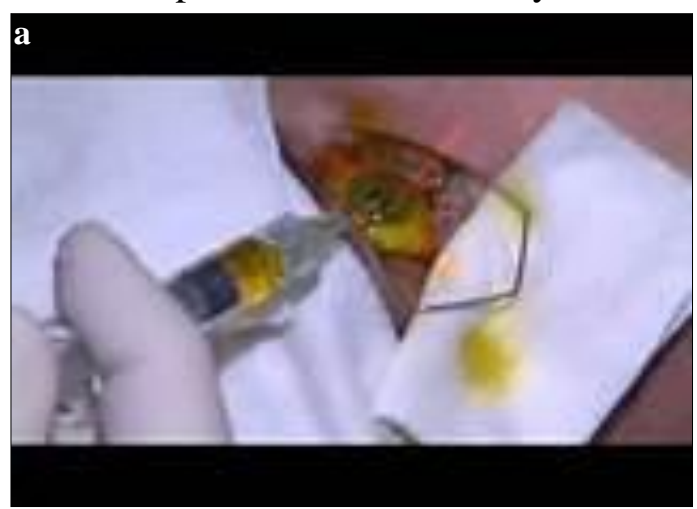

Figure (3) a. application of riboflavin before UVA,

\subsubsection{Operative details}

After applying the eyelid speculum, a specially formulated riboflavin solution, Ricrolin TE (Carleton Optical, Chesham Buckinghamshire HP5 2BD, UK), was used. the epithelium was not removed. All procedures were performed under topical anesthesia, instilled twice for $2 \mathrm{~min}$ before the procedure, fig. $(3-a, b)$.

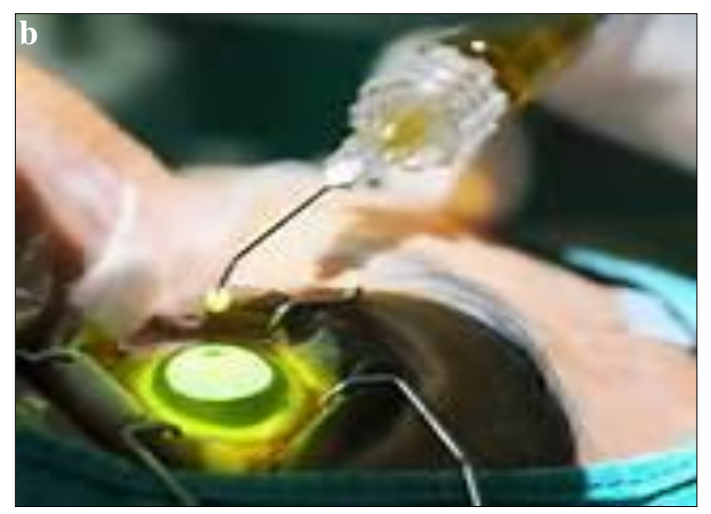

application of riboflavin during UVA.

Two enhancers, trometamol and sodium EDTA, were added to this solution every 3 min for 20-30 min until the stroma was completely penetrated. 


\subsubsection{Postoperative medication and follow-up}

A topical combination of steroids and antibiotic drops five times daily for 1 week, tapered to four times daily for next week, and then two times daily for next 2 weeks was prescribed. Lacrimal substitutes were administered four times daily for 4-6 weeks. Patients were followed-up for 6 months postoperatively. Follow-up was

\subsubsection{Statistical analysis}

Categorical variables are described as numbers and percentages, and continuous variables are described as mean and SD. The $\chi 2$-test was used to compare categorical variables, and the t-test was used to compare continuous variables. Continuous variables were tested for normal distribution using

\section{Results}

The incidence of postoperative pain was lowered by about $30 \%$ in the transepithelial group compared with the epioff group in the first postoperative week, fig. (4). The incidence of postoperative corneal haze was lowered by about $50 \%$ in the trans-epithelial group compared with the epi-off group in the first postoperative week, fig. (5). Collagen CXLs formed in the classic debridement technique were much deeper in the stroma compared with the trans-epithelial technique, fig. (6). After 6 months postoperatively, optical coherence tomographic analyses of the corneal stroma showed the demarcation line at a depth of 320-340 nm of corneal thickness in group I, whereas in group II the demarcation line

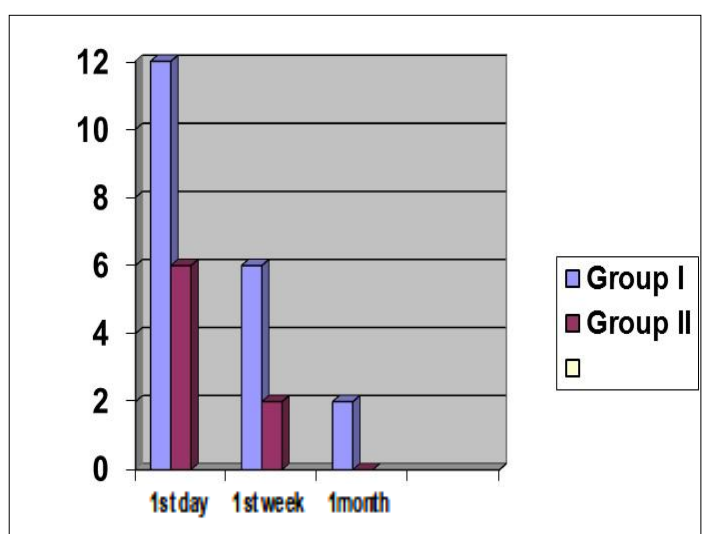

Figure (4) Postoperative pain in $1^{\text {st }}$ day, $1^{\text {st }}$ week and $1^{\text {st }}$ month in the 2 groups carried out at 1 week, 3 , and 6 months postoperatively; patients were examined for their UCVA, BSCVA, refraction, intraocular pressure, corneal function, and any complaints. Also, topography, keratometry, corneal astigmatism, and pachymetry were carried out using Pentacam.

the Kolmogorov-Smirnov test and Q-Q plots. A two-tailed $\mathrm{P}$ value less than 0.05 was considered statistically significant. All analyses were performed using SPSS 20.0 software (IBM SPSS Statistics, New York, NY, USA).

did not exceed $120 \mathrm{~nm}$ of corneal thickness. Corneal pachymetry was carried out using an oculus Pentacam. In group I, none statistically significant corneal thinning was detected after 3 and 6 months with a mean pachymetry score (thinnest) of 444.78 +38.75 , fig. (7). Although there was statistically non-significant corneal thinning in Group II after 3 months, there was some regression in the form of corneal thinning by around 5-10 m after 6 months in 20\% of cases with a mean pachymetry score (thinnest) of $430.78+25.75$. We found statistically significant differences between groups I and II as shown, figs (8 and 9) and tab. (1).

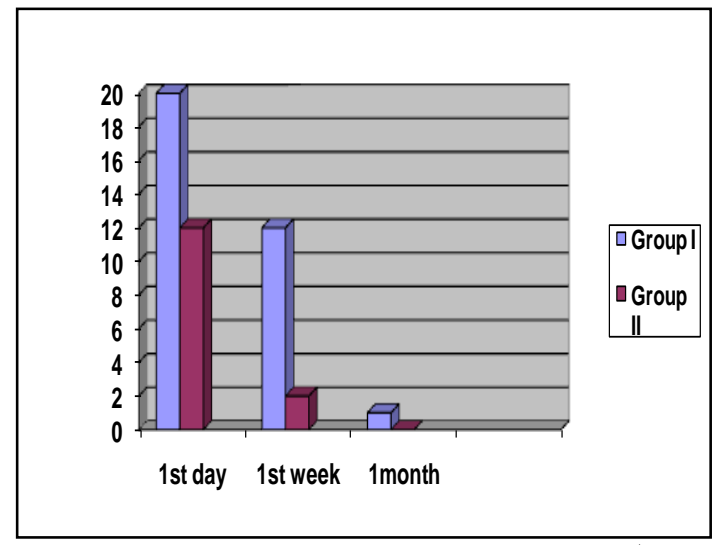

Figure (5) Postoperative corneal haze in $1^{\text {st }}$ day, $1^{\text {st }}$ week and $1^{\text {st }}$ month 

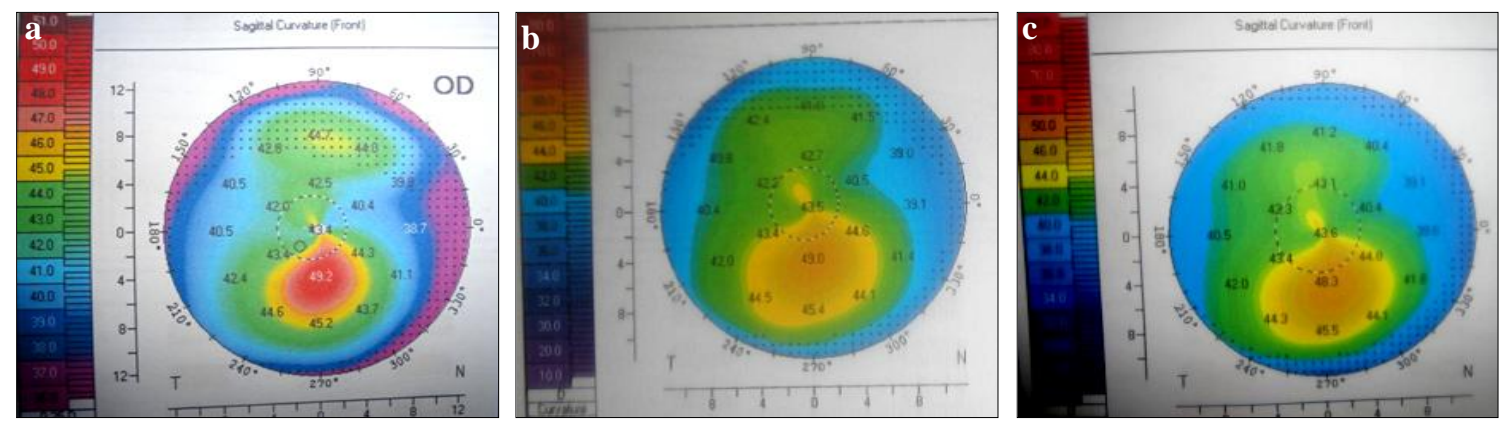

Figure (6) Photos of case 6 of group I showing the changes in corneal topography $\underline{\mathbf{a}}$. at preoperative, $\underline{\mathbf{b}}$. at 3 months, $\underline{\mathbf{c}}$. at 6 months.
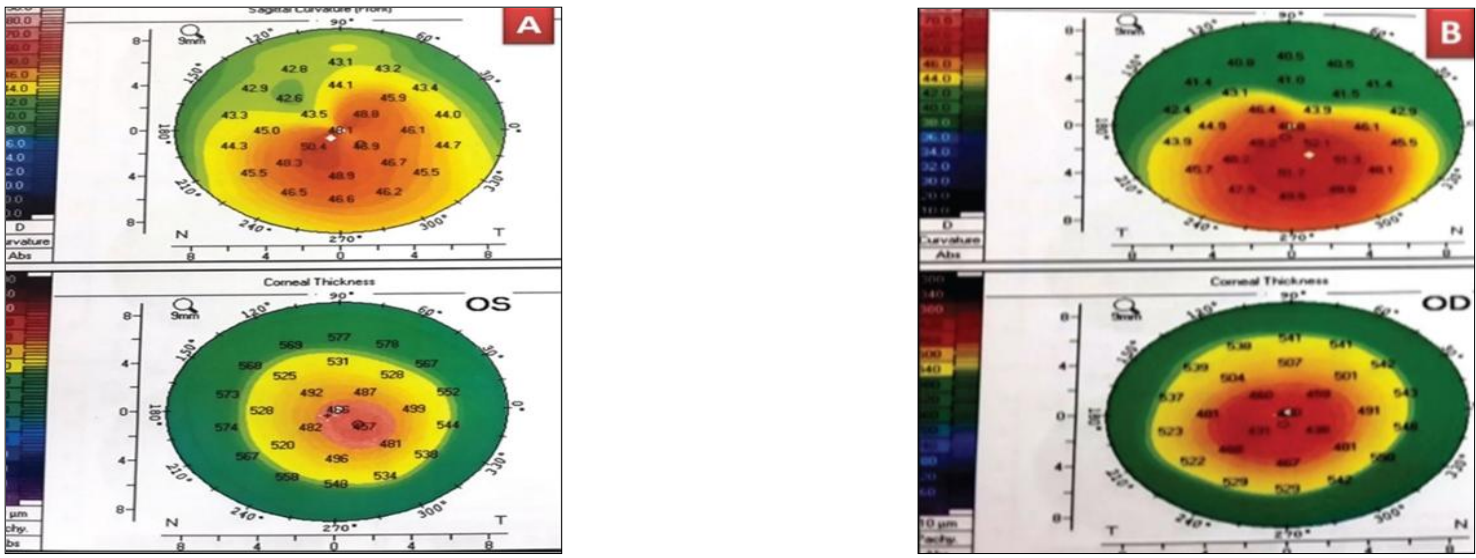

Figure (7) Case number 8 a $\cdot$ preoperative, $\underline{\mathbf{b}}$. postoperative 6 months epi off CXL.

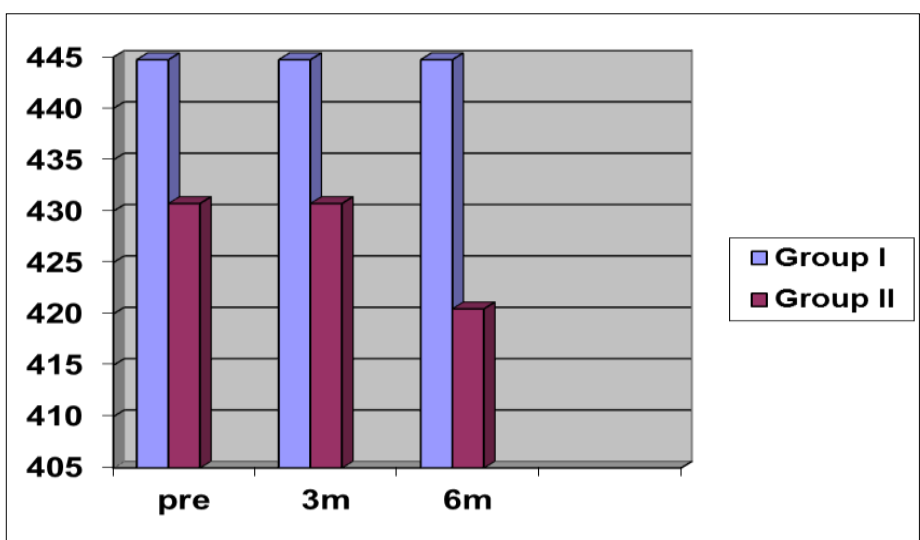

Figure (8) Pachymetry (thinnest) changes preoperative, postoperative 3 and 6 months in the 2 groups.
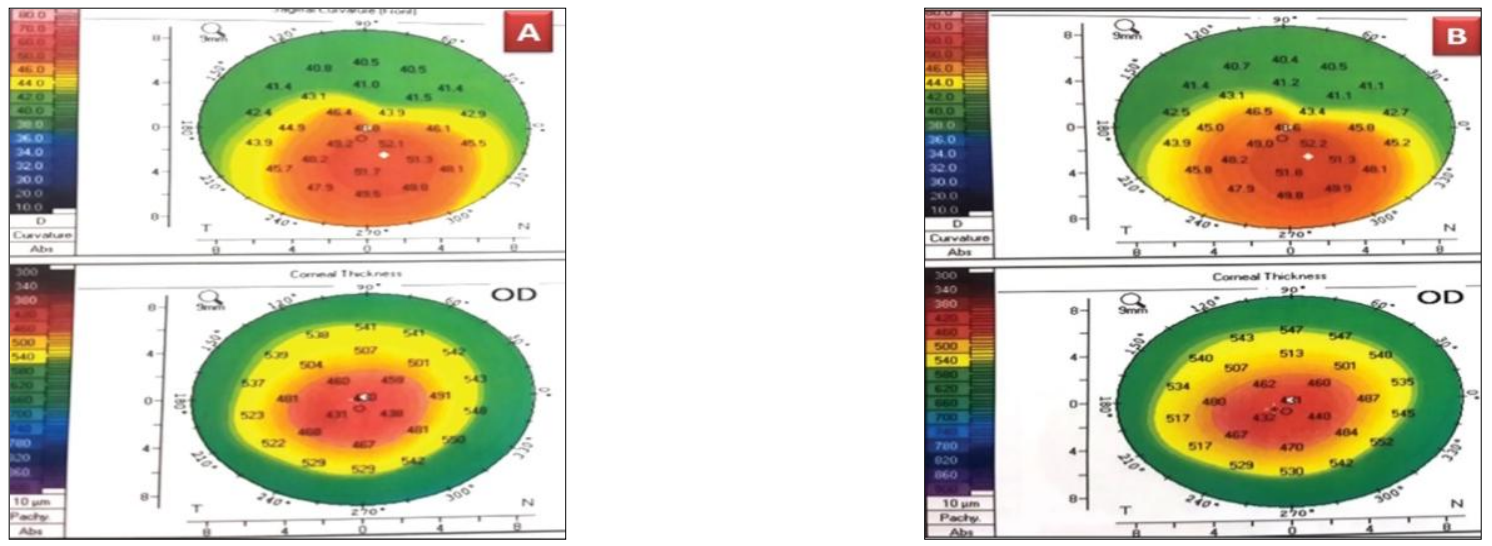

Figure (9) Case number 18 a. preoperative, $\underline{\mathbf{b}}$. postoperative epi on CXL. 
Table (1) Comparison of preoperative mean uncorrected visual acuity, best spectacle-corrected visual acuity, Kmax, corneal astigmatism, and pachymetry score between the two groups.

\begin{tabular}{|l|c|c|c|}
\multicolumn{1}{c}{ Pre-operative: } & Group I & Group II & P. value \\
\hline Mean Kmax & $50.15 \pm 3.50$ & $49.15 \pm 3.50$ & $0.372^{\text {ns }}$ \\
\hline Mean UCVA & $0.1 \pm 0.14$ & $0.2 \pm 0.2$ & $0.075^{\mathrm{ns}}$ \\
\hline Mean BSCVA & $0.3 \pm 0.2$ & $0.4 \pm 0.1$ & $0.053^{\mathrm{ns}}$ \\
\hline Mean Astigmatism & $-5.06 \pm 2.97 \mathrm{D}$ & $-4.06 \pm 2.97$ & $0.294^{\mathrm{ns}}$ \\
\hline Mean pachymetry & $\mathbf{4 5 0} \pm \mathbf{3 0}$ & $\mathbf{4 4 0} \pm \mathbf{3 0}$ & $\mathbf{0 . 2 9 8}^{\mathrm{ns}}$ \\
\hline
\end{tabular}

BSCVA, best spectacle-corrected visual acuity; UCVA, uncorrected visual acuity. $t$-test used.

\subsection{Visual and refractive outcomes}

\subsubsection{Uncorrected distance visual acuity}

The mean preoperative UCVA was $0.1 \pm 0.14$, whereas the mean postoperative UCVA at 3 months was $0.15 \pm 0.1$, showing an improvement by a mean of 0.05 Snellen line. At 6 months, the mean postoperative UCVA was $0.2 \pm 0.05$, showing an improvement by a mean of 0.1 Snellen line. Comparisons between the mean preoperative UCVA and the mean postoperative UCVA at 3 and 6 months are shown in tab. (2). There was a highly significant difference between the results of UCVA preoperatively and the results of UCVA at 3 and 6 months postoperatively. The mean preoperative BSCVA was $0.3 \pm 0.15$, whereas the mean

postoperative BSCVA at 3 months was $0.4 \pm 0.1$, showing an improvement by a mean of 1 Snellen line. At 6 months, the mean BSCVA was $0.5 \pm 0.1$, thus showing an improvement by a mean of 1 Snellen line. Comparisons between the mean preoperative BSCVA and the mean postoperative BSCVA at 3 and 6 months are shown in tab. (3). There was a significant difference between the results of the BSCVA preoperatively and the results of the BSCVA at 3 and 6 months postoperatively. A minimal difference was detected between 3 and 6 months of follow-up, denoting that patients mostly stabilize beyond this time, fig. (10).

Table (2) Showing the changes in UCVA throughout the study

\begin{tabular}{|c|c|}
\hline Mean preoperative UCVA & $0.1 \pm 0.14$ \\
\hline Mean postoperative UCVA at 3 months & $0.15 \pm 0.1$ \\
\hline Mean postoperative UCVA at 6 months & $0.2 \pm 0.05$ \\
\hline
\end{tabular}

$\boldsymbol{U C V A}$, uncorrected visual acuity.

Table (3) Showing the changes in BSCVA throughout the study and its significance.

\begin{tabular}{|c|c|}
\hline Mean preoperative BSCVA & $0.3 \pm 0.15$ \\
\hline Mean postoperative BSCVA at 3 months & $0.4 \pm 0.1$ \\
\hline Mean postoperative BSCVA at 6 months & $0.5 \pm 0.1$ \\
\hline
\end{tabular}

BSCVA, best spectacle-corrected visual acuity.

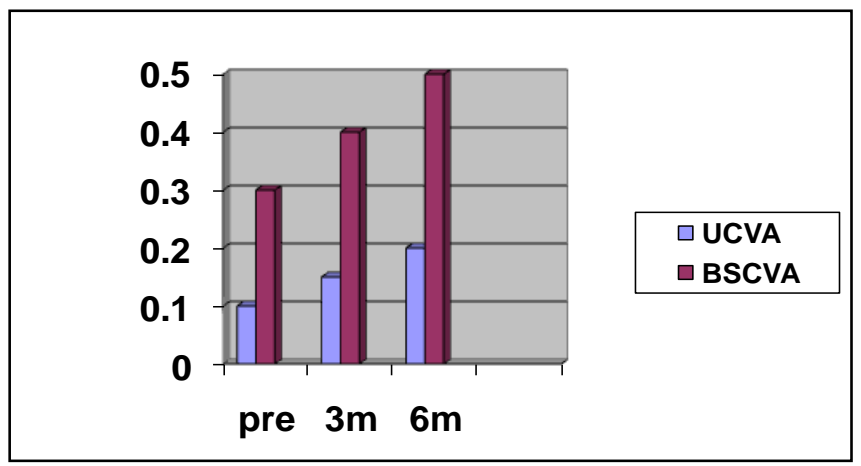

Figure (10) BSCVA, best spectacle-corrected visual acuity; UCVA, uncorrected visual acuity.

\subsubsection{Uncorrected visual acuity in each group}

The mean preoperative UCVA for group I was $0.1 \pm 0.14$, whereas the mean postoperative UCVA at 3 months was $0.18 \pm 0.05$ and at 6 months $0.2 \pm 0.05$, with 
a mean improvement of 0.13 Snellen lines. The mean preoperative UCVA for group II was $0.118 \pm 0.05$, whereas the mean postoperative UCVA at 3 months was $0.2 \pm 0.05$ and at 6 months $0.26 \pm 0.18$, with a mean improvement of 0.14 Snellen lines. The differences between the mean preoperative UCVA and the mean postoperative UCVA at 3 and 6 months between the two groups are shown in tab. (4).

Table (4) Comparing preoperative and postoperative mean UCVA and BSCVA readings at 6 months among the 2 study groups.

\begin{tabular}{|l|c|c|c|}
\hline & Group I & Group II & P. value \\
\hline Mean preoperative UCVA & $0.1 \pm 0.14$ & $0.118 \pm 0.05$ & $0.591^{\mathrm{ns}}$ \\
\hline Mean postoperative UCVA at 6 m & $0.2 \pm 0.05$ & $0.26 \pm 0.18$ & $0.159^{\mathrm{ns}}$ \\
\hline Mean preoperative BSCVA & $0.3 \pm 0.15$ & $0.218 \pm 0.204$ & $0.155^{\text {ns }}$ \\
\hline Mean postoperative BSCVA & $\mathbf{0 . 6} \pm \mathbf{0 . 1 1}$ & $\mathbf{0 . 4 8} \pm \mathbf{0 . 1 8}$ & $\mathbf{0 . 0 1 5}$ \\
\hline
\end{tabular}

T-test used BSCVA, best spectacle-corrected visual acuity, UCVA, uncorrected visual acuity. $P<0.05$, * Significant difference

\subsubsection{Best spectacle-corrected visual acuity in each group}

The mean preoperative BSCVA for group I was $0.3 \pm 0.15$, whereas the mean postoperative BSCVA- at 3 months was $0.45 \pm 0.1$ and at 6 months $0.6 \pm 0.11$. The mean preoperative BSCVA for group II was $0.318 \pm 0.204$, whereas the mean postoperative BSCVA at 3 months was $0.35 \pm 0.1$

\subsection{Pentacam findings}

\subsection{1. $\mathrm{K}$ readings preoperatively and postoperatively for the two groups}

The topographic analysis carried out using Pentacam showed a mean preoperative Kmax of $48.06 \pm 5.05 \mathrm{D}$ in group I. The mean postoperative Kmax at 3 months was $47.1 \pm 5.6$ with a reduction of $-0.96 \mathrm{D}$. The mean postoperative Kmax at 6 months was $44.5 \pm 3.2$, thus showing an increase in the mean reduction by $3.56 \mathrm{D}$. In group II, the and at 6 months $0.48 \pm 0.18$. The differences between the mean preoperative bestcorrected visual acuity (BCVA) and the mean postoperative BCVA at 6 months between the two groups, using the analysis of variance single factor test, are shown in tab. (4).

Table (5) k Readings pre and postoperative of two groups:

\begin{tabular}{|c|c|c|c|}
\hline & Group I & Group II & P. value \\
\hline Mean preoperative Kmax & $48.06 \pm 5.05$ & $47.2 \pm 4.30$ & $0.565^{\mathrm{ns}}$ \\
\hline Mean post-operative Kmax at 3months & $47.1 \pm 5.6$ & $47.1 \pm 4.5$ & $0.999^{\mathrm{ns}}$ \\
\hline Mean postoperative Kmax at 6 months & $44.5 \pm 3.2$ & $46.9 \pm 4.2 \mathrm{D}$ & 0.049* \\
\hline
\end{tabular}

T-test used ed. $* \mathrm{P}<0.05$, significant difference.

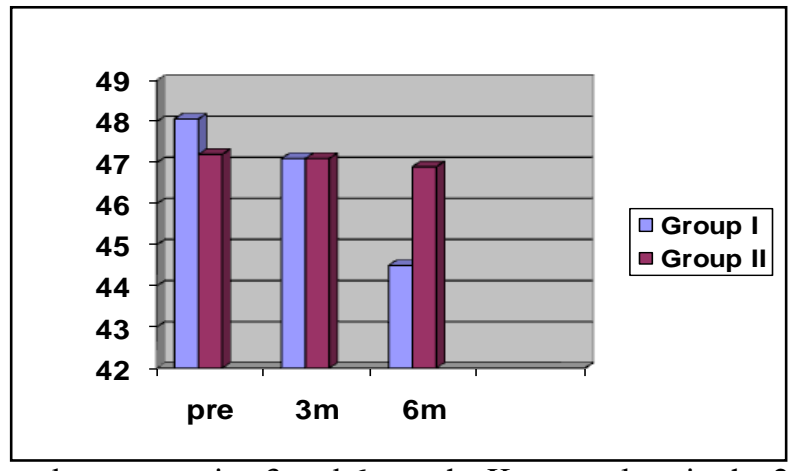

Figure (11) Preoperative and postoperative 3 and 6 months $\mathrm{K}$ max values in the 2 groups. 


\section{Discussion}

The basic clinical outcomes of the epi-off CXL group seem to follow a reproducible time course after treatment. In general, VA and corneal steepness worsen somewhat at 1 month, and resolution to baseline occurs by $\sim 3$ months, with improvement thereafter. This similar to the clinical time course for corneal haze; thus, stromal and epithelial healing responses to the epi-off CXL appear to continue over months, concomitant with the changes in clinical outcomes. An important criterion to evaluate the effects of CXL in our study was to evaluate its effect on corneal thickness. Non significant corneal thinning was recorded after 3 and 6 months; this means that corneal thickness was almost back to its original value. At 6 months postoperatively, pachymetry in group I showed that there was no statistically significant corneal thinning, which meant that there was no progression of keratoconus. On the other hand, in group II after 6 months there was some regression in the form of corneal thinning by around $5-6 \mu \mathrm{m}$ in $20 \%$ of cases. These results are similar to the study conducted by Caporossi et al., who also recorded an initial non significant decrease in the pachymetry values of all patients, and then no statistically significant difference in corneal thickness was observed after 3 months of follow-up [5]. On the contrary, Vinciguerra et al., found that there was a significant decrease in corneal thickness after 12 months of follow-up [6]. Also, Wollensak et al., evaluated the clinical usefulness of riboflavin/UVA-induced collagen crosslinking in progressive keratoconus and reported a cessation of keratoconus progression in all treated eyes but in 16 eyes $(70 \%)$ regression with a reduction of the maximal keratometry readings by 2.01 diopters and of the refractive error by 1.14 diopters was found [7]. In the epi-on group, study, at the last follow-up examination 6 months postoperatively, the UCVA was $0.26+0.18$ with a mean improvement of 0.14 Snellen lines. The BSCVA was $0.48+0.18$ with a mean improvement of
0.262 Snellen lines. The mean value of postoperative astigmatism at 6 months was $-3.96+1.4$ with a hyperopic shift of +0.04 D. The mean postoperative Kmax at 6 months was $46.5+4.2 \mathrm{D}$, an increase in mean reduction by $-0.8 \mathrm{D}$. Similarly, Stojanovic et al., evaluated the efficacy and safety of epi-on CXL and observed significant improvement of uncorrected and corrected distance visual acuity at 12-month. None of the eyes lost lines of CDVA, while $27.4 \%$ of the eyes gained 2 or more lines. Mean spherical equivalent decreased by $0.74 \mathrm{D}$, and mean cylindrical reduction was 1.15 D [8]. Also, Rossi et al., reported significant improvement in uncorrected corrected visual acuity after epi-on CXL treatment with significant improvement in topographic outcomes, spherical error, and spherical equivalent at month 12 [9]. By comparing postoperative topographic outcomes of the two groups at 6 months, we found that Kmax in group I had an increase in the mean reduction by $2.31 \mathrm{D}$ and group II had an increase in the mean reduction by $0.8 \mathrm{D}$. Soeters et al., in his study to compare the clinical effects and safety of transepithelial CXL to epi-off CXL in progressive keratoconus noticed that average $\mathrm{K}$ max was stable at all visits in the transepithelial group, while after epioff CXL a significant flattening was demonstrated from the 3-month follow-up onwards and the trend over time in $\mathrm{K}$ max flattening was significantly different between the groups [10]. In contrast, Kocka et al., in their comparison between transepithelial CXL and epi-off CXL detected that Mean maximum cone apex curvature (apical K) increased from $51.62 \pm 5 \mathrm{D}$ to $53.70 \pm 5.49 \mathrm{D}$ in transepithelial group, and decreased from $52.02 \pm 4.07 \mathrm{D}$ to $51.22 \pm 3.51$ in the epi-off CXL group at the end of 12 months with significant difference between two groups [11]. At the 6-month postoperative pachymetry (thinnest location) in group I, a non significant corneal thinning was recorded, which means that there was no progression of keratoconus. On the other hand, in group II after 6 months there 
was some sort of regression in the form of corneal thinning by about $5-10 \mu \mathrm{m}$ in $20 \%$ of cases. Cifariello et al., compared the outcomes of CXL epi-on and CLX epi-off on forty eyes from 32 patients with progressive keratoconus after 2 years of followup. They noticed significant improvement in visual function and unchanged Keratometry in both groups. The mean corneal thickness showed a significant red-uction in both groups. However, Fibrotic reaction and activated keratocyte were more commonly recorded in CLX epi-off Group than in CXL epi-on Group [12].

\section{Conclusion}

Corneal CXL treatment is a relatively new, revolutionary, minimally invasive procedure aiming at stabilization of keratoconus. The creation of new chemical bonds (CXLs) between collagen fibrils at the corneal stroma by the combined use of riboflavin and ultraviolet irradiation can result in an increase in the biomechanical stability of the corneal tissue, halting the progression of the keratoconus. Data of our study as well as other studies showed that both the epi-off CXL and the epi-on CXL were effective in stabilizing the progression of keratoconus at least for 6 months (the duration of follow-up in our study). However, the epi-off CXL appeared to be much more effective in improving the refractive BCVA and topographic outcomes in keratoconic eyes compared with the epi-on CXL. On the other hand, our study found that the epi-on CXL had less postoperative complications such as pain and corneal haze and was more comfortable for patients.

\section{References}

1. Weissman B., Yeung K., Keratoconus. 2007; http://www.emedicine. com.

2. Caporossi A., Mazzotta C., Baiocchi S., et al. Long-term riboflavin ultraviolet A corneal collagen cross-linking for keratoconus in Italy. Am J Ophthalmol. 2010; 149: 585-593.

3. Kymionis G, Diakonis V, Kalyvianaki M., et al. One-year follow-up of corneal confocal microscopy after corneal crosslinking in patients with post laser in situ keratomileusis ectasia and keratoconus. Am J Ophthalmol. 2009; 147: 774-778.

4. Bethke W. Cross section of cross-linking. Rev Ophthalmol. 2013; 20: 32.

5. Caporossi A., Baiocchi S., Mazzotta C., et al. Parasurgical therapy for keratoconus by riboflavin ultraviolet type A rays induced crosslinking of corneal collagen: Preliminary refractive results in an Italian study. J Cataract Refract Surg. 2006; 32: 837-845.

6. Vinciguerra P., Albè E., Trazza S., et al. Refractive, topographic, tomographic and aberrometric analysis of keratoconic eyes undergoing corneal cross-linking. Ophthalmology. 2009; 116: 369-378.

7. Wollensak G., Spoerl E., Seiler T. Riboflavin/ultraviolet-A-induced collagen crosslinking for the treatment of keratoconus. American J of Ophthalmology. 2003; 135 (5): 620-627.
8. Stojanovic A., Chen X., Jin N., et al. Safety and efficacy of epithelium-on corneal collagen cross-linking using a multifactorial approach to achieve proper stromal riboflavin saturation. $J$ of $\mathbf{O p h}$ thalmology. 2012; 2012: 498435.

9. Rossi S., Orrico A., Santamaria C. et al. Standard versus trans-epithelial collagen cross-linking in keratoconus patients suitable for standard collagen crosslinking. Clinical Ophthalmology (Auckland, NZ). 2015; 9: 503.

10. Soeters N., Wisse R., Godefrooij D. et al. Transepithelial versus epitheliumoff corneal cross-linking for the treatment of progressive keratoconus: A randomized controlled trial. American J of Ophthalmology. 2015; 159 (5): 821-828.

11. Kocak I., Aydin A., Kaya F., et al. Comparison of transepithelial corneal collagen crosslinking with epitheliumoff crosslinking in progressive keratoconus. J Francais d'Ophtalmologie. 2014; 37 (5): 371-376.

12. Cifariello F., Minicucci M., Di Renzo F., et al. Epi-Off versus Epi-on corneal collagen cross-linking in keratoconus patients: A comparative study through 2-year follow-up. J of Ophthalmology. 2018; 2018:4947983. 